\title{
ZU BEETHOVENS BRIEF AN DIE UNSTERBLICHE GELIEBTE (PSYCHOLOGISCHE UND MEDIZINISCHE ASPEKTE)
}

\author{
Vlastimil ¿ e r mák (Brno)
}

Das Entstehungsdatum von Beethovens Brief an die sogenannte unsterbliche Geliebte war seit jeher ein Gegenstand historisch, künstlerisch und nicht zuletzt auch chronologisch motivierter polemischer Erwägungen.

Trotzdem herrscht bei der Beantwortung dieser Frage auch heute noch keine Einigkeit, man hat die verschiedensten Vermutungen geäußert und manchmal sogar unbewiesene und sensationslüsterne Schlüsse gezogen.

Beim Studium von Beethovens Korrespondenz aus dem Jahre 1801, der von manchen Autoren noch immer fest angenommenen Entstehungszeit des Briefs an die unsterbliche Geliebte, konnte ich keine Anzeichen dafür finden, daß diese Jahreszahl richtig ist. Im Gegenteil: die psychologische und stilkritische Untersuchung dieser Korrespondenz führte mich zur Uberzeugung, daß der umstrittene Brief im Jahr 1801 gar nicht entstehen konnte. Als einzige Alternativmöglichkeit ist das Jahr 1812 ins Auge zu fassen.

Selbstverständlich kann die vorliegende Studie die Erkenntnisse und Daten, die verschiedene Autoren zu dieser Frage gesammelt haben, ${ }^{1}$ nur

${ }^{1}$ Aus der umfangreichen Literatur seien angeiuhrt: Kalischer A. Ch., Die unsterbliche Geliebte Beethovens Giulietta Guicciardi oder Therese Brunswick? Dresden 1891. - Jachimecki Z., Das richtige Datum des Briefes L. v. Beethovens »an die unsterbliche Geliebte». Zeitschrift der Internationalen Musikgesellschaft, 9 (1907-1908), 349 ff. - Thomas-San-Galli, W. A., Beethoven und die unsterbliche Geliebte: Amalie Sebald, Goethe, Therese Brunsvik und anderes. München 1910, Ders., Die unsterbliche Geliebte Beethovens Amalie Sebald, Lösung eines viel umstrittenen Problems. Halle 1909. - Unger M., Auf Spuren von Beethovens unsterblicher Geliebten. Musikalisches Magazin, Heft 37. Langensalza 1911. - Leitzmann A., Beethoven's zweiter Brief an die unsterbliche Geliebte eine Fälschung? Zeitschrift der Internationalen Musikgesellschaft, 12 (1910-1911), 350 ff. - La Mara (M. Lipsius), Beethoven und die Brunsviks. Leipzig 1920, $80 \mathrm{ff.}$ - Frimmel Th., Beethoven-Handbuch 2. Leipzig 1926, 347 ff. - Hevesy A., Beethoven, Vie intime, Paris 1926. - Sonneck O. G. Th., The Riddle of the Immortal Beloved. A Supplement to Thayer's Life of Beethoven. New York 1927. - Rolland R., Les Aimées de Beethoven. Paris 1949. - Kaznelson S., Beethovens ferne und unsterbliche Geliebte. Zurüch 1954. - Major E., A shalhatatlan kedves«. Adatok a Beethoven kutatáshoz, Romain Rolland két ismeretlen levelével kapcsolatban ("Die unsterbliche Geliebte«, Beitrag zur Forschung über Beethoven nach zwei unbekannten Briefen von Romain Rolland). - Steichen D., Beethoven's Beloved. New York 1959. - 
von einem anderen Blickwinkel aus ergänzen. In diesem Zusammenhang sei vor allem an die Arbeit des führenden tschechischen Musikwissenschaftlers Jan Racek gedacht, der sich mit diesem Thema ausführlich befaßt.? Meine Untersuchung stützt sich auf die beiden Briefe aus Beethovens' Korrespondenz vom 29. Juni und 16. November 1801, die also in relativ kurzem Abstand geschrieben wurden. Es waren ja gerade diese beiden Schreiben, die den Vertretern der Behauptung, der Brief an die unsterbliche Geliebte sei nicht im Jahr 1812, sondern im Jahr 1801 entstanden, als Vergleichsmaterial dienten. Eine irrige Ansicht, die in der tschechischen Beethoven-Literatur namentlich von Anton Klodner und Vladimir Karbusický ${ }^{3}$ vertreten wird.

Beethoven schreibt am 29. Juni 1801 seinem Freund Wegeler, einem der wenigen Menschen, denen der Meister seine privaten Angelegenheiten anvertraut. Nach dem Datum fällt dieser Brief in die Zeit knapp von der folgenden, allerdings nur hypothetischen Abreise Beethovens nach Pieštany im Jahr 1801, wie Klodner und Karbusický behaupten.4

In diesem Schreiben heißt es unter anderem: "Da riet mir ein medizinizcher asinus das kalte Bad für meinen Zustand, ein gescheiterer das lauwarme Donaubad: das tat wunder, mein Bauch ward besser, mein Gehör blieb oder ward noch schlechter«. 5 Beethoven hat sich also der erwähn. ten Heilprozedur im Donauwasser unterzogen, seine Befriedigung darüber

Schmidt-Görg J., Wasserzeichen in Beethoven-Briefen, Beethoven-Jahrbuch 1961/4, Bonn, 1966. - Ders., Wer war »die M." in einer nichtigen Aufzeichnung Beethovens? Beethoven Jahrbuch 1961/64, Bonn, 1966, 75.

2 Beethovens Brief an die sogenannte unsterbliche Geliebte und seine genaue Datierung behandelt Jan Racek in folgenden Buch- und Zeitschriftenveröffentlichungen: L. v. Beethoven, Studie o životě a díle (L. v. Beethoven, Studie über Leben und Werk). Praha 1929. Malá edice Odeonu, Bd. 16. - Beethoven a naše země (Beethoven und unsere Länder), Sammelschrift L. v. Beethoven. Praha 1942, $23 \mathrm{ff}$. - Beethoven, rüst hrdiny a bojovnika (Beethoven - Werdegang eines Helden und Kämpfers). Praha 1955, 2. Aufl. 1956, besonders $119 \mathrm{ff}$. Beethoven a češké země (Beethoven und die Böhmischen Länder). Brno 1964, Opera universitatis Purkynianae Brunensis - Facultas philosophica 91. - Wann und wo entstand Beethovens Brief an die sogenannte "Unsterbliche Geliebte"? Zum Problem Beethoven in den böhmischen Ländern. Mitteilungen der Kommission für Musikforschung der Oest. Akademie der Wissenschaften, Nr. 21, Wien 1972, 206 f. -Wann und wo entstand Beethovens Brief an die "Unsterbliche Geliebte«? Festschrift für Ernst Hermann Meyer zum sechzigsten Geburtstag, herausgegeben von G. Knepler. Leipzig 1973, 265-272.

3 Klodner A., Beethovenov "List nesmrtel'nej milenke" (Beethovens Brief an die "Unsterbliche Geliebte»). Hudební rozhledy 15 (1962), 693. - Ders., List nesmrtel'nej milenke a Slovensko (Der Brief an die unsterbliche Geliebte und die Slowakei). Ebda. 16 (1961), 889 f. - S. auch Karbusický V., Beethovnuiv list. "An die unsterbliche Geliebte" a jeho hudebni dílo (Beethovens Brief "An die unsterbliche Geliebte« und sein musikalischen Werk). Praha 1969.

${ }^{4}$ Franz Gerhard Wegeler (1765-1848), Gatte der Eleonore Breuning, bis zum Jahr 1794 Professor an der Bonner Universität, schliesslich praktischer Arzt in Koblenz. In Bonn verkehrte er mit Beethoven bis zur U̇bersiedlung des Komponisten nach Wien, wo auch Wegeler vorubergehend (1794-1796) lebte. Im Jahr $1838 \mathrm{gab}$ er zusammen mit Ries Biographische Notizen über L. v. Beethovens heraus; seine Erinnerungen sind die wichtigste Quelle für Beethovens Bonner Zeit. Wegeler gehörte zu den vertrautesten Freunden des Meisters.

${ }^{5}$ Leitzmann A., Beethovens Brief, Leipzig 1912, 21. 
geäußert und es nicht unterlassen, Wegeler zu benachrichtigen. Leider ist es nicht möglich, den Ort zu bestimmen, an dem Beethoven die Donaubäder nahm, es konnte sich um die verschiedensten Stellen am Donaustrom handeln, in der Nähe Wiens oder weiter von der Hauptstadt entfernt.

Der Brief aus dem Juni 1801 ist aber auch in anderer Hinsicht interessant. Beethoven spricht zwar ziemlich ausführlich über das »lauwarme Donaubad«, verliert aber kein Sterbenswörtchen über die angeblich geplante Badekur in Pieštany, die den Andressaten doch sicherlich auch als Arzt interessiert hätte. Und dabei soll der Komponist nur wenige Tage später die weite Reise angetreten und den Brief an die unsterbliche Geliebte am 6. und 7. Juli 1801 gerade in der Slowakei (Pieštany-Dolná KrupáKorompa) verfaßt haben! ${ }^{6}$ Oder sollte Beethoven seinen Freund Wegeler absichtlich über seine Reise ins Heilbad im Unklaren gelassen haben? Das ist kaum anzunehmen. Fügen wir noch hinzu, daß der Komponist in seinem Brief zwar anführt "-Schreibe mir jetat öfter;" aber merkwürdigerweise dem Freund seine unmittelbar bevorstehende neue Anschrift in Pieštany gar nicht mitteilt. ${ }^{7}$ Man wird wohl zugeben, daß das Fehlen einer jeglichen Notiz über die geplante Abreise nach Pieštany in diesem Briel schon an und für sich ernste Zweifel an Klodners und Karbusickýs Argumentation für einen Aufenthalt Beethovens in Pieštany während der Badesaison 1801 erwecken muß.

Der zweite Brief an Wegeler stammt vom 16. November 1801 und erhärtet unsere Ansicht, daß der Brief an die unsterbliche Geliebte nicht im Jahr 1801 geschrieben wurde. Man lese doch nur jene Stelle aufmerksam: "Diese Veränderung hat ein liebes, zauberisches Mädchen hervorgebracht, die mich liebt und die ich liebe. Es sind seit zwei Jahren wieder einige selige Augenblicke, und es ist das erstemal, daß ich fühle, daß - Heiraten glïcklich machen könnte. Leider ist sie nicht von meinem Stande - und jetzt - könnte ich nun freilich nicht heiraten - ich muß mich nun noch wacker herumtummeln. ${ }^{8}$

Ganz im Gegensatz zu dem in Frage stehenden Brief erwägt hier Beethoven seine Lebenslage sehr objektiv und erkennt sachlich an, daß er dieses Mädchen - abgesehen von dessen hohem Stand - aus Existenzgründen gar nicht heiraten könnte, ohne diese Frage dann weiter zu zergliedern. Der nüchterne Inhalt der Mitteilung steht in klarem Widerspruch

- Dolń́ Krupá (Alsó Korompa) ist ein slowakisches Dorf $35 \mathrm{~km}$ südwestlich von Pöstyén (Pieštany), $10 \mathrm{~km}$ nördlich von Tyrnau (Trnava) und $50 \mathrm{~km}$ nordöstlich von Pressburg (Bratislava). A. Klodner und V. Karbusický sprechen die unbegrindete Vermutung aus, der Brief an die unsterbliche Geliebte sei nicht in Teplice, sondern wahrscheinlich in der Slowakei (Pieštany-Dolná Krupá-Korompa) geschrieben worden. Die beiden Autoren beweisen auch durchaus hypothetisch, Beethoven habe sich im Jahr 1801 in Pieštany einer Badekur unterzogen Diese Behauptung hat bei uns J. Racek ỉberzeugend widerlegt, unter anderem in der Studie Znovu o nesmrtelné milence (Abermals über die unsterbliche Goliebte), Hudební rozhledy XVI, 1963, Nr. 15, 615 f., weiter in der Studie Odpo véd Antonu Klodnerovi (Antwort an Anton Klodner), Hudební rozhledy XVI, 1963, Nr. 22, 933.

7 Leitzmann, 1. c., 23.

- Leitzmann, 1.c., 25. 
zum romantisch-poetischen Schwung von Beethovens berühmtem Brief, in dem des Meisters Geist in den Fernen schwebt und seine Liebe als Himmelsgebäude sieht; jubelnde Begeisterung, vollste geistige und körperliche Hingabe - dies alles spricht von einer ganz anderen Einstellung zur Liebe als sie unser Zitat aus dem Brief an Wegeler vom Herbst des Jahres 1801 verrät.

Der Brief vom 16. November 1801 ist aber noch durch einem anderen Umstand interessant: man findet dort abermals kein einziges Wort über den Erfolg von Beethovens Kur in Pieštany, die ja angeblich im Jahr 1801 stattgefunden hat, im selben Jahr also, in dem der Brief an die unsterbliche Geliebte entstanden sein soll.

Nun noch einige psychologische Aspekte, die sich aus dem in Frage stehenden Brief Beethovens ergeben. Unter der Voraussetzung, daß dieser Brief tatsächlich eine konkrete Person betraf, bietet die Stelle "Ist es nicht ein wahres Himmelsgebäude, unsre Liebe? - aber auch so fest, wie die Feste des Himmels ${ }^{9}$ guten Grund für die Vermutung, daß eine solche Liebe kaum das Ergebnis einer kurzen oder gar vorübergehenden Bekanntschaft sein konnte. Sie mußte sicherlich auf eine längere Dauer zurückblicken, wurde von dem Leben wohl mehrmals auf die Probe gestellt und hatte alle Prüfungen bestanden. Auch diese zwingende Vermutung scheint mit der durchaus hypothetischen Ansicht unvereinbar zu sein, Beethoven habe den Brief an die unsterbliche Geliebte im Jahr 1801 ge schrieben.

Wir werden sehen, wie der Meister in dem umstrittenen Brief die Aussichten seiner Liebesbeziehung auf die Waagschale legt. Schon der gesamte Habitus dieses Schreibens spricht eher dafür, daß er vom reifen Beethoven stammt, also nicht im Jahr 1801 geschrieben wurde. In diesem Zusammenhang sind folgende Zeilen aus dem Brief vom 7. Juli beachtenswert: "In meinen Jahren jetat bedürfte ich einiger Einförmigkeit, Gleichheit des Lebens....10 So mochte der vierzigjährige Beethoven sprechen, der reife leiderfahrene Mann, nicht aber der dreißigjährige Stürmer und Dränger, der sich damals ohnehin in einer genialischen Unausgeglichenheit gefiel, die beinahe $\mathrm{zu}$ einem Zug seines moralischen Profils geworden wäre. Im Jahr 1801 hätte der junge Beethoven diese Worte kaum gebraucht, die ujbrigens im Zusammenhang mit dem hochfliegend ernsten Ton des Briefes etwas paradox wirken mußten.

Hier drängt sich allerdings auch die Vermutung auf, das Schreiben, das als "Brief an die unsterbliche Geliebte" in die Literatur eingegangen ist, sei überhaupt an keine bestimmte Frau gerichtet worden. Konnte der Meister nicht in höchster Bewegung der Sinne, zwischen schmerzlichen Liebeserinnerungen und getäuschten Liebeshoffnungen schwankend, diesen Brief an eine erträumte Geliebte sadressiertı haben, konnte dieser Brief nicht eine Art Sublimierung seiner Sehnsucht gewesen sein?

Gegen diese Vermutung spricht allerdings der konkrete Inhalt mancher Briefstellen. Beethoven unterstreicht beispielsweise den Umstand, der

- Leitzmann, 1. c., 88.

18 Leitzmann, 1. c., 88. 
Brief sei mit jenem Bleistift geschriben, den ihm die Geliebte geschenkt habe, er beschreibt den Verlauf seiner beschwerlichen Reise, die Art und Weise, wie er nach dem Bestimmungsort kam; und die Worte, er müsse schließen, damit der Brief rechtzeitig ankomme, seine Freude auf das baldige Wiedersehen, das alles weist eher auf eine bestimmte Adressatin als auf eine literarische Fiktion hin. Die Beziehung Beethovens zu seiner Angebeteten wird, im ganzen gesehen, in einer so selbstverständlichen Weise erwogen, daß die Zweifel an der oben geäußerten Vermutung berechtigt erscheinen.

In diesem Zusammenhang wäre noch ein weiterer, nämlich der medizinische Aspekt ins Treffen zu führen, der ebenfalls nicht auf einen Kuraufenthalt Beethovens im Jahr 1801 schließen läßt.

Vorausgeschickt sei, daß sich Beethoven kaum entschlossen hätte, sein Gehörleiden gerade in Pieštany zu kurieren, einem Bad, dessen Heilmöglichkeiten dem Indikationsregister von Erkrankungen des Gehörs oder der Verdauungsorgane gar nicht entsprechen. In Pieštany pflegte man nämlich seit jeher vor allem Krankheiten des Bewegungsapparats, also rheumatische Beschwerden, pathologische Zustände der Bindegewebe, Gelenke und Muskel usw. zu heilen.

Die Badekur hätte also in Beethovens Fall keinen spezifischen Effekt gezeitigt und konnte höchstens der Erholung oder Zerstreuung dienen. Für einen solchen Aufenthalt Beethovens fehlen aber überzeugende Quellen.

Wie sah der Gesundheitszustand des Komponisten um das Jahr 1801 eigentlich aus? Beethoven litt damals an Gehörschwierigkeiten. Seine Hypacusis (Schwerhörigkeit) stand noch im Anfangsstadium, in dem es sicher auch zu zeitweiligen Remissionen der Krankheitssymptome gekommen ist. Bekanntlich fand sich Beethoven damals mit seinem noch leidlich guten Gehör im täglichen Leben halbwegs zurecht. Jedenfalls fühlte er keine Gefahr einer dauernden Verschlechterung seines Zustands. Die bereits auftauchenden Verdauungsstörungen konnten auf einen früher überstandenen Abdominaltyphus zurückgehen. Uber andere ernste Gesundheitsbeschwerden bieten die Quellen keine Berichte. Ganz anders war die Lage im Jahr $1812 \ldots$

Beethoven mußte damals bereits eine Periode der Gehörverschlimmerung erleben, die an Taubheit grenzte. Außerdem nahmen die Verdauungsbeschwerden bedenklich $\mathrm{zu}$ (Colitis catarrhalis, Pankreatitis et Hepatitis chronica). Begreiflicherweise hatten diese Krankheiten die Entstehung einer Leberschrumpfung mit allen ihren Komplikationen zur Folge, die am 26. März 1827 Beethovens Leben ein Ende bereiteten. ${ }^{11}$

Zum Charakter von Beethovens Ohrenkrankheit wäre zu erwähnen, daß es sich um eine Schwerhörigkeit perzeptorischen Typs handelte, bei deren Ätiologie irgendwelche Komplikationen ernster Krankheitszustände eine Rolle spielen konnten, die der Komponist überwunden hatte. Komplizie-

11 Schweisheimer W., Beethovens Krankheiten. Zu Beethovens 150. Geburtstag am 17. XII. 1920. Sonderabdruck aus der Münchener medizinischen Wochenschrift 1920, Nr. 51, 1473-75. Ders., Beethovens Leiden, ihr Einfluss auf sein Leben und Schaffen. München 1921. 
rende Faktoren mochten auch von einer früheren Blatternerkrankung hergerührt haben, mit den Folgen einer toxischen Neuritis und Verletzung des Gehöranalysators; darüber hinaus lassen sich nicht einmal Komplikationen einer überstandenen akuten Hepatitis (infektiösen Leberentzündung) und anderer, damals noch unbekannter Viruserkrankungen ausschließen. Die häufigen Kopfschmerzen lassen auf die Möglichkeit irgendeiner überstandenen Komplikation schließen, die die Hirnhaut betraf (in lokaler oder diffuser Form).

Im Rahmen des perzeptorischen Typs von Beethovens Schwerhörigkeit kommt auch ein otosklerotischer Proze $\beta$ in Betracht, mit anderen Worten: es konnte sich um jenen Typ der Otosklerose handeln, bei dem es zuerst $\mathrm{zu}$ degenerativen Strukturänderungen des Cortischen Organs im inneren Ohr kommt; dieser Typ kann sich später mit dem sogenannten Steigbügeltyp verbinden und es entsteht die perzeptorisch-konduktive Form der Schwerhörigkeit. Darauf sollte die Tatsache hindeuten, daß Beethoven angab, hohe Töne zum Unterschied zu niedrigeren Frequenzen nicht zu hören. Die perzeptorische Störung mit Lädierung des Cortischen Organs äußert sich nämlich gerade im Hörverlust höherer Frequenzen. Auch das als quälend empfundene Ohrensausen ist für diesen Gehörfehler typisch. Otosklerose tritt häufig als Folge einer erblichen Belastung auf. Immerhin ist es problematisch, vielleicht sogar unmöglich, jene Krankheitskomponente mit Sicherheit ausfindig $\mathrm{zu}$ machen, die zu Beethovens Taubheit führte; es fehlen nämlich die entsprechenden Belege, vor allem ärztliche Gutachten aus seiner Lebenszeit.

Der Aufenthalt in Bad Teplice in den Jahren 1811 und 1812 war wohl einer der letzten Versuche Beethovens, das unheimliche Fortschreiten der Zerstörung seines Gehörs aufzuhalten oder wenigstens zu verlangsamen. Der Meister hatte Hufelands Handbuch kennengelernt und dort erfahren, man heile in Bad Teplice Störungen des Stoffwechsels, Gicht, Rheumatismus, Nervenkrankheitein, ja sogar Erkrankungen der Sinnesorgane, vor allem des Gehörsinns u.a. ${ }^{12}$ Auch Beethovens persönlicher Arzt Johann Malfatti ${ }^{13}$ hatte ihm den Aufenthalt empfohlen.

Aber nicht einmal die Kuren im westböhmischen Bad konnten den Lauf der verhängnisvollen Krankheit hemmen. Immerhin hat sich der zweite Teplitzer Aufenthalt des Meisters in die Musikhistorie eingeschrie-

${ }^{12}$ Christian Wilhelm Hufeland (1762-1836) wirkte zehn Jahre lang als praktischer Arzt in Weimar und verkehrte dort mit Goethe, Herder, Schiller und Wieland. Im Jahr 1793 wurde er Professor zu Jena, wo er mit grossem Erfolg Makrow biotik vortrug, im Jahr 1809 Professor für Pathologie und Therapeutik zu Berlin. Aus Hufelands Handbuch schöpfte Beethoven Informationen liber die Eignung der Teplitzer Badekur für sein Gehörleiden.

13 Johann Malfatti (1775-1859), ein Wiener Arzt italienischer Herkunft, behandelte Beethoven in den Jahren 1809-1815. Malfatti empfahl dem Komponisten im Jahr 1812 einen Kuraufenthalt im westböhmischen Bad Teplice. Dies geht aus einem Schreiben hervor, das Beethoven im Jahr 1812 aus Wien an Josef Freherrn von Schweiger, den Kämmerer Erzherzog Rudolfs, richtete. Dort heisst es: "Malfatti will durchaus, dass ich nach Teplitz soll, das ist mir nun gar nicht lieb. (Kastner 248, Brief Nr. 345). 
ben - dort entstand nämlich der Brief an die unsterbliche Geliebte vom 6. und 7. Juli 1812.

Zum Schluß will ich meine Folgerungen kurz zusammenfassen. Vor allem bestätigt der Inhalt des im Juni 1801 an Wegeler gerichteten Schreibens Beethovens Entschluß, in naher Zukunft Bad Pieštany zu besuchen, in keiner Weise. Auch im zweiten Schreiben an Wegeler aus dem November desselben Jahres vermißt man jede Erwähnung oder gar Würdigung eines Kuraufenthaltes in diesem Bad. Zudem war ja Beethovens Ohrenleiden im Jahr 1801 noch nicht so weit fortgeschritten, daß es dringend eine Badekur erfordert hätte. Utberdies umfaßt die Indikation der in Pieštany heilbaren Krankheiten weder Gehörleiden noch Verdauungsbeschwerden. Und schließlich widersprechen einander Beethovens eigene Worte über Liebe und Ehe im Schreiben vom Juni des Jahres 1801 an Wegeler und in seinem Brief an die unsterbliche Geliebte.

Diese kurzen Bemerkungen lassen wohl erkennen, daß die Ausführungen Klodners und Karbusickýs reichlich problematisch sind - diese Forscher bemühen sich nämlich mit Hilfe eines stilkritischen Vergleichs der dreißigjährigen Beethoven mit seinem in Frage stehenden Brief die identische Herkunft aus dem Jahr 1801 nachzuweisen. Nach dem Inhalt der angeführten beiden Schreiben aus dem Jahr 1801 läßt sich aber ganz im Gegenteil glaubwürdig klarstellen, daß der Brief an die unsterbliche Geliebte in dieser Zeit gar nicht entstehen konnte. Infolgedessen erhärtet sich die Utberzeugung, Beethoven habe diesen Brief im Juli des Jahres 1812 im westböhmischen Bad Teplice geschrieben.

\section{POVZETEK}

Avtor podrobno analizira v svoji študiji omenjeno Beethovnovo pismo z medicinskega in psihološkega vidika. Neodvisno od dosedanjih raziskav, ki so so nanašale na to vprašanje, prihaja $\mathrm{k}$ sklepu, da je cit. pismo nastalo $\mathrm{v}$ dneh 6 . in 7. julija $1812 \mathrm{v}$ zahodnočeških zdraviliščih. $\mathrm{S}$ tem potrjuje rezultate, do katerih sta se $v$ svojem raziskovanju prikopala Jan Racek (Brno) in Josef Schmidt-Görg (Bonn), zavrača pa naziranja, ki sta jih imela $\mathrm{v}$ tej zvezi Anton Klodner in Vladimir Karbusický. Njegovi izsledki dopolnjujejo glasbeno zgodovinske, ki jim Čermak dodaja nova spoznanja o omenjenem, doslej tako zapletenem in spornem vprašanju. Do svojih sklepov je prišel avtor pričujočega sestavka z raziskova-' njem psihične in somatične patografije Beethovnove osebnosti ter preučevanjem vsebinskega karakterja Beethovnove korespondence iz razdobja 1801-1812, ki jo $\checkmark$ tej zvezi kritično konfrontira. Poleg drugega razpravlja tudi o vzrokih, ki so pri Beethovnu privedli do gluhosti, pa še o drugih patoloških faktorjih, ki so znani za skladateljevo zdravstveno napeto življenje. S pomočjo kriterijev sodobne medicinske vede strokovno kritično vrednoti medicinske diagnoze, ki so bile za Beethovna postavljene oz. dane $\mathrm{v}$ času njegovega življenja in še po smrti. Znanstveno poskuša precizirati še posamezne patološke sindrome mojstrovíh bolezni. S tega aspekta tudi pokaže na vsebinsko polno Beethovnovo pismo, ki je predmet tega sestavka, in ugotavlja natancen datum tega znamenitega beethovenskega dokumenta. 